Meta

Journal des traducteurs

Translators' Journal

\title{
Le plus et le moins-disant culturel du texte juridique. Langue, culture et équivalence
}

\section{Jean-Claude Gémar}

Volume 47, numéro 2, juin 2002

Traduction et terminologie juridiques

URI : https://id.erudit.org/iderudit/008006ar

DOI : https://doi.org/10.7202/008006ar

Aller au sommaire du numéro

\section{Éditeur(s)}

Les Presses de l'Université de Montréal

ISSN

0026-0452 (imprimé)

1492-1421 (numérique)

Découvrir la revue

Citer cet article

Gémar, J.-C. (2002). Le plus et le moins-disant culturel du texte juridique. Langue, culture et équivalence. Meta, 47(2), 163-176.

https://doi.org/10.7202/008006ar

\section{Résumé de l'article}

Le texte juridique est sans doute un des plus marqués culturellement. Il reflète la complexité d'une société, dont les institutions et le vocabulaire sont l'expression d'une culture parfois multimillénaire. La common law et le système civiliste n'en présentent pas moins de nombreuses similitudes équivalences ou quasi-équivalences - qui reposent sur un certain nombre d'universaux communs à tous les systèmes juridiques. Mais ces systèmes présentent aussi de grandes différences qui tiennent à des écarts culturels parfois considérables. On peut parler ici d'intraduisibilité ou de quasi-intraduisibilité de termes porteurs d'une notion étrangère au système et à la culture d'arrivée. La réponse du traducteur variera en conséquence du " plus-disant culturel » (constitution, chartes) au « moins-disant » (contrat). La fonction et la destination du texte en détermineront le mode d'expression. 


\title{
Le plus et le moins-disant culturel du texte juridique. Langue, culture et équivalence
}

\author{
JEAN-CLAUDE GÉMAR \\ ETI, Genève, Suisse
}

\begin{abstract}
RÉSUMÉ
Le texte juridique est sans doute un des plus marqués culturellement. II reflète la complexité d'une société, dont les institutions et le vocabulaire sont l'expression d'une culture parfois multimillénaire. La common law et le système civiliste n'en présentent pas moins de nombreuses similitudes - équivalences ou quasi-équivalences - qui reposent sur un certain nombre d'universaux communs à tous les systèmes juridiques. Mais ces systèmes présentent aussi de grandes différences qui tiennent à des écarts culturels parfois considérables. On peut parler ici d'intraduisibilité ou de quasiintraduisibilité de termes porteurs d'une notion étrangère au système et à la culture d'arrivée. La réponse du traducteur variera en conséquence du «plus-disant culturel» (constitution, chartes) au «moins-disant» (contrat). La fonction et la destination du texte en détermineront le mode d'expression.
\end{abstract}

\begin{abstract}
Legal texts abound in culturally loaded terms expressing a society's complex institutions and vocabulary generated by cultures stretching back thousands of years. The common law and civil law systems bear numerous common features that are more or less equivalent and based upon a number of universals common to all legal systems. They also differ in many aspects as they present great cultural gaps that render translation of certain terms hazardous or even impossible since they may convey notions unknown to the target system and culture. The way the translator will address this problem shall vary according to the cultural load borne, in any given system, by the phrases and terms, from the highest (charter, constitution, statute) to the lowest (contract, regulation). The translator's response to this challenge will be governed by the legal functions of the text and its target readers.
\end{abstract}

\section{MOTS-CLÉS/KEYWORDS}

traduction, langue de spécialité, culture, texte juridique, équivalence

Le langage du droit occupe une place particulière dans l'imaginaire du traducteur. Pour le commun des mortels, qu'il intimide, le droit est un domaine tellement vaste et complexe que seuls des initiés peuvent s'y risquer. Il est vrai que le droit s'exprime de bien des façons au sein d'une même langue et d'un même système, et plus différemment encore d'une langue et d'un système à l'autre. Aussi, lorsqu'il passe par le tamis de la traduction, le texte de droit subit-il des transformations étonnantes.

On s'interrogera alors sur la réussite de l'opération traduisante et sur l'équivalence, réelle ou supposée, des deux textes. Lorsqu'il s'agit de traduire un texte «pragmatique» ou non - le transfert de son contenu culturel est toujours une source de difficulté pour le traducteur. Porteur de notions souvent chargées d'histoire et de 
traditions, le texte juridique, de par sa charge culturelle, est particulièrement représentatif de ce type de difficulté. Posons alors la question: le traducteur juridique peut-il produire un texte d'arrivée satisfaisant à la fois dans la lettre et dans l'esprit pour un destinataire d'une culture différente de celle du texte de départ? Et cette "équivalence», au terme de l'interprétation qu'en fera un tribunal, par exemple, peut-elle aller jusqu'à garantir des effets juridiques équivalents dans les deux textes? La question - ou plutôt: ces questions - est vaste et innombrables ou presque sont les réponses à y apporter, selon qu'elles seront données par un juriste, un linguiste, un philosophe, un sociologue ou... un traducteur.

La première partie de mon texte sera consacrée à la question de la langue de spécialité en situation de traduction. Le facteur culturel y occupera une grande place, même si je ne ferai que survoler un problème dépassant de loin la seule traductologie, discipline qui se situe néanmoins au cœur de cette problématique. Ensuite, la question de la langue de spécialité qu'est le langage du droit sera brièvement envisagée, car ce sujet est désormais sinon épuisé du moins bien connu des jurilinguistes. En revanche, la traduction de la langue de spécialité, la juridique en particulier, reste un sujet inépuisable et il importe de bien comprendre ce que l'on doit entendre par «texte juridique» avant de parler de ses rapports à la traduction. En deuxième partie, il ne restera plus qu'à aborder le "pont aux ânes» de la traduction, soit la question de l'équivalence. Quoique rebattu, ce sujet reste un problème qui est loin d'être résolu, en traductologie générale comme en traduction spécialisée.

Appliqué à la traduction juridique, cet idéal d'équivalence reste un terrain à défricher, toujours aussi vague malgré les apports récents des traductologues. Il suffit de poser la question de la comparaison ou de la distinction à faire entre traduire et adapter pour mesurer la complexité de la notion d'équivalence. Elle garde tout son mystère, qui est celui du langage, des langues naturelles et de la "parole» qu'elles produisent, donc du sens et des significations qui en découlent.

\section{Langue, culture et traduction}

Produit social — qui en douterait, désormais? - , la langue est le creuset où se sculpte l'histoire d'un peuple. Les mots qui la composent et le discours qui l'exprime traduisent les valeurs d'un patrimoine commun. En d'autres termes: une «culture» — si tant est que l'on puisse s'entendre sur le sens à donner à ce terme gigogne, que linguistes et traductologues ont aussi défini. Pour le linguiste Émile Benvéniste par exemple, la culture est «le milieu humain, tout ce qui, par-delà l'accomplissement des fonctions biologiques, donne à la vie et à l'activité humaines, forme, sens et contenu» (1966: 30). Peter Newmark, le traductologue, voit dans la culture: «[...] the way of life and its manifestations that are peculiar to a community that uses a particular language as its means of expression» (1988: 94). Or, pour Claude Hagège, agir sur les langues revient à agir sur «les cultures elles-mêmes» (1985: 204), ce qui ne saurait laisser indifférent le traducteur. Langue et culture sont donc indissociables.

Aussi, pour saisir toute la portée d'un terme recouvrant une notion aussi riche, faudrait-il remonter aux sources de la civilisation et de ses mythes fondateurs (Dumézil 1995), et convoquer simultanément l'anthropologie (Lévi-Strauss 1949) la sociolinguistique et la traductologie (Nida 1996), voire, pour ce qui relève spécifiquement du texte juridique, l'anthropologie juridique (Rouland 1991, Vanderlinden 
1996). On trouvera chez ces auteurs un traitement et un éclairage de la question plus dense et plus fouillé que celui d'un propos annexe dans le cadre d'un article.

À qui douterait encore que la culture est consubstantielle à la langue, l'exemple du Canada montre une étroite interaction des aspects linguistiques, culturels et sociopolitiques (Plourde 2000) qui en fait un phénomène rare, et même un cas d'espèce. La traduction est au cœur de ce débat historique. Beaucoup d'idées reçues circulent sur les rapports entre culture et traduction. On pense, et cette intuition est souvent avérée, que plus une culture est éloignée de celle du traducteur, plus celui-ci aura de la difficulté à la faire passer dans son texte d'arrivée. Il n'en faut pas pour autant sous-estimer les obstacles que présentent les langues et cultures «germaines». Comme l'observe Iso Camartin, l'étranger, c'est d'abord et avant tout le voisin (Camartin 1996: 24). La proximité, voire la cohabitation des langues et des systèmes ne garantit nullement le «passage» d'un fait culturel (Sparer 1979).

La «force» d'une langue résiderait-elle alors, comme on semble le penser communément, dans sa capacité à "repousser l'étranger»(Berman 1984: 26) ? À entendre certains discours prônant la "pureté » linguistique, le retour à l'essence, au "génie» de telle langue, on pourrait le croire. La véritable force d'une langue, toutefois, ne viendrait-elle pas plutôt, comme le croyait Gothe, de sa capacité à «dévorer» l'étranger? Mais en le dévorant, il en restera toujours quelque chose. Voir, par exemple, les principales langues européennes et leurs emprunts, aussi nombreux qu'anciens, aux autres langues au cours de leur évolution et la marque indélébile, pour une fois du français sur l'anglais le plus courant - par exemple: stop! Ce qui a pu pousser Iso Camartin (Camartin 1996), à propos du rhéto-romanche, à croire que même si toute culture (et langue) est profondément enracinée dans sa région, le fragment — la langue et la culture locales — ne saurait pour autant ignorer l'étranger — le tout: le voisin, le monde — au risque de se scléroser. L'exemple du Canada, et tout particulièrement du Québec, où la langue française, au contact de l'anglais, a évolué de façon étonnante depuis la « révolution tranquille» qui a marqué les années soixante (Plourde 2000: 232-319), pourrait faire illusion. Pour une réussite — toute relative néanmoins - combien d'échecs?

Le dilemme que nous pose Octavio Paz à propos de la compréhension des autres, n'est-il pas au fond du problème devant lequel le traducteur est quotidiennement placé, problème lié à l'ethnocentrisme (Berman, 1984) dont feraient preuve les empires (ou, de nos jours, les superpuissances) culturels : «La compréhension des autres est un idéal contradictoire: elle nous demande de changer sans changer, de devenir autres sans cesser d'être nous-mêmes ${ }^{1}$.» (Paz, 1983: 31)?

Mais c'est à un tout autre ordre de compréhension qu'il faut penser lorsque, avant de passer à la traduction d'un texte, il s'agira de le comprendre. Quelle que soit la complexité des opérations intervenant dans le processus conduisant à la compréhension d'un texte - phénomène hors de mon propos ici —, il s'agit pour le traducteur de reconnaître au premier coup d'œil le domaine dont relève ledit texte.

\section{La langue de spécialité et le langage du droit}

Quel que soit le domaine, le texte réalisé, avec tous ses éléments de sens apparents et sous-jacents, sa syntaxe et sa stylistique particulières, est le produit d'une langue de spécialité (ou «langue spécialisée »: Lerat 1995: 20) mise en discours. En simplifiant 
la question à l'extrême ${ }^{2}$, le texte juridique présente trois caractéristiques qui le distinguent des autres: il s'agit d'un texte normatif disposant d'un style et d'un vocabulaire particuliers.

«Au commencement était la règle» nous dit Jean Carbonnier (1995: 96). La caractéristique première du droit est sa nature normative: le législateur fixe la règle. Ensuite, le droit exprime cette norme de diverses façons, par la loi, le jugement et l'acte (juridique). Chacun de ces textes est rédigé selon des formes que l'on ne peut confondre entre elles (le style d'une loi n'est pas celui d'un jugement, lequel diffère de celui d'un acte), avec un vocabulaire distinctif (par ex.: action en répétition de l'indu, contrat synallagmatique, créancier chirographaire).

Le langage du droit, enfin, est loin d'être uniforme, même s'il est parfois univoque dans ses termes. Contrairement à d'autres domaines, son registre est des plus étendus. Il va du plus pragmatique - le texte contractuel, par exemple - au plus esthétique, voire mystique (Nida, 1982: 261). Dans cette dernière catégorie peuvent entrer des textes aussi chargés de symboles que la Déclaration des droits de l'Homme et du Citoyen (1789), le Code Napoléon (1804) ou certains textes de doctrine dont la valeur littéraire est reconnue.

Autant de particularités qui font du droit, lorsqu'il s'agit de traduire un texte juridique, un domaine singulier voire paradigmatique, compte tenu des difficultés que présente l'opération traduisante en l'espèce. Le droit est un des domaines les plus culturels, donc singuliers, qui soient. Il remonte aux sources de la civilisation, de chaque langue et de la culture qu'elle porte. De plus, le droit est un phénomène local et, comme Candide ou l'Optimisme (Voltaire, 1759) nous l'a plaisamment montré, il franchit difficilement les frontières nationales.

Le langage du droit d'un pays exprime en conséquence et au plus haut degré la charge historique d'une notion, d'une institution, par exemple: rule of law, due process (of law) ou reasonable man / person, trust ou consideration des pays de common law; «arrêt», «État de droit», «immeuble», «nue-propriété» ou «quasi-délit» des Français; "société libre et démocratique» des Canadiens, etc. Et que dire du mot «droit» lui-même, si l'on compare ce terme à l'anglais law ou à l'allemand Recht? Leur traduction dans une autre langue, si tant est qu'elle existe (cf. common law?), rend-elle justice à la richesse de la notion dont ces termes sont porteurs?

\section{Langage du droit et traduction}

Les spécialistes s'entendent plus ou moins sur les moyens dont dispose le traducteur. Vinay et Darbelnet (1958) ont notamment défini sept "procédés» de traduction: emprunt, calque, traduction littérale, transposition, modulation, équivalence, adaptation. De nombreux autres traductologues ont proposé, depuis, d'autres moyens et façons d'aborder le texte à traduire pour tenter d'atteindre l'équivalence. Je ne retiendrai ici les procédés de Vinay et Darbelnet qu'à la seule fin d'illustrer quelques exemples de traduction de termes ou d'expressions juridiques possibles.

1. L'emprunt = Trust, Common Law, Equity

2. Le calque $=$ Tribunal de première instance / Court of first instance [JUDIT]

3. Traduction littérale $=$ Connaître d'une requête / Take cognizance of an action [JUDIT] 

4. Transposition
5. Modulation

\section{6. Équivalence}
7. Adaptation

$=$ in consideration of / en contrepartie de

$=$ Statuer, toutes affaires cessantes / postpone all other cases and give a decision [JUDIT]

$=$ siéger dans une affaire $/$ sit in a case [JUDIT] Rule of Law $=$ Rechtstaat $=$ État de droit

$=$ statut d'une personne morale de droit privé / instruments constituting and regulating a legal person governed by private law [JUDIT]

Selon le contexte et la situation de travail, on pourrait sans doute se satisfaire de ces solutions, proposées par les dictionnaires, lesquels abondent en solutions. Certaines sont bonnes, d'autres moins. Or, le langage du droit véhicule des notions, des institutions et des procédures qui sont tellement propres à chaque langue et culture juridiques que l'on ne peut les transposer telles quelles d'une langue et d'un système à un autre sans risquer à tout moment l'impropriété, le contresens, voire le non-sens juridiques (Crépeau 1993; Sacco, 1999: 169; Vanderlinden 1998; Viau 2000). Aussi la traduction juridique, tout particulièrement entre le français et l'anglais, pose-t-elle un problème particulièrement aigu dans la mesure où il ne s'agit pas simplement de passer d'une langue à une autre, mais d'un système à un autre, si différents l'un de l'autre (Didier, 1990). Mais avant d'aborder la question du texte juridique et de sa traduction, il importe de définir ce que l'on doit entendre par «texte juridique».

\section{Qu'est-ce qu'un «texte juridique»?}

Même si la question peut paraître saugrenue, les avis sont néanmoins partagés et une certaine confusion règne dans les esprits. Laissons aux linguistes la définition du mot «texte», qui est à prendre ici dans son sens courant d'écrit, et voyons plutôt ce que laisse entendre l'adjectif «juridique».

Selon le Petit Robert ( $\mathrm{V}^{\circ}$ juridique 2.), est juridique ce qui «a rapport au droit», puisque cet adjectif qualifie ce substantif. Une telle définition couvre un large champ sémantique et annonce de ce fait une typologie des textes juridiques étendue. Pour le profane peu averti, la notion de texte juridique recouvre généralement celle du texte parajuridique ou à coloration juridique qu'est, par exemple, l'article d'un quotidien consacré à la chronique judiciaire ou le texte du règlement interne affiché sur la porte d'une chambre d'hôtel. Il suffira que ledit texte contienne quelques termes vaguement assimilables au vocabulaire du droit pour qu'il soit taxé de juridique.

Ce problème de polysémie est propre aux langues naturelles, et l'adjectif, dans la hiérarchie des signifiants, ne vient qu'au troisième rang, après le verbe et le substantif. Remplacé par le substantif «droit», juridique revêt un sens plus précis, celui que les juristes reconnaissent dans la définition de Gérard Cornu: "Est juridique tout discours qui a pour objet la création ou la réalisation du droit» (Cornu, 1990: 21). Ici, la typologie se réduit aux textes qui créent ou réalisent du droit, ce qui restreint cette catégorie aux producteurs de textes que sont le législateur, le juge et les gens de loi.

Il s'ensuit qu'en droit, le texte juridique sera porteur d'une charge culturelle plus ou moins lourde selon que l'on aura affaire, dans le système romano-germanique qu'est le français par exemple, à une production du législateur (constitution, charte, loi), du juge (décision de justice) ou, entre autres, du notaire (acte juridique en général et contrat en particulier). Du plus- au moins-disant culturel, en somme. Sans 
négliger les exceptions possibles: par exemple, un arrêt d'une Cour d'appel ou de la Cour suprême portant sur une question hautement «culturelle» (la langue...), une loi ou une disposition portant sur une question d'ordre très pratique.

Mais ce plus- ou moins-disant revêt-il la même signification pour tous les lecteurs? Quelle que soit l'intention de son auteur, nous dit Norbert Rouland, une fois écrit «le texte "parle" tout autant dans la façon dont le reçoit celui qui le lit que dans l'intention de son auteur» (Rouland, 1991: 34). Or, le lecteur n'est pas toujours à même de saisir, en sus de sa portée juridique, la charge culturelle d'un terme ou d'une expression du langage du droit - par ex.: rule of law (R.U.) ou «société libre et démocratique» (Can.). Je distinguerai à cet égard, à l'instar de nombreux juristes, quatre grandes catégories de lecteurs, du plus inculte au plus savant: 1) le lecteur profane peu ou prou lettré; 2 ) le lecteur lettré ( «l'honnête homme ou femme» du XVIII ${ }^{\mathrm{e}}$ siècle) ; 3 ) le juriste praticien (les gens de loi); 4) le savant (théoricien, comparatiste, entre autres). Selon que le texte juridique sera lu par l'une ou par l'autre personne de telle ou telle catégorie, sa teneur juridique sera plus ou moins bien saisie, mais le fondement culturel (ou socio culturel), sauf exception, en échappera en quasi-totalité au lecteur de la première catégorie ou en partie au lecteur de la deuxième catégorie, voire de la troisième.

À l'évidence, le lecteur profane d'un texte (juridique) rédigé dans une langue étrangère éprouvera une difficulté encore plus grande, alors que le comparatiste, mieux au fait des différences que comporte le système étranger par rapport au sien, en mesurera mieux que les autres le poids et la portée sémantiques.

Transposé à la situation du traducteur, et non plus du simple lecteur, le texte juridique à traduire pose nombre de problèmes (Sacco, 1999: 169), dont celui des choix que fera le traducteur.

Pour le traducteur, aux obstacles juridiques que présentent des notions de droit non concordantes entre les systèmes viennent s'ajouter des contraintes linguistiques incontournables, dont la lisibilité du texte destiné à tel public en particulier n'est pas la moindre.

\section{Contextes juridiques, contraintes linguistiques}

Les choses se compliquent encore d'autant pour le traducteur si l'on veut préciser qu'il peut être appelé à œuvrer dans trois, voire quatre grandes catégories différentes de contexte culturel, linguistique comme juridique.

En partant, encore une fois, du plus simple pour aller au plus complexe, on trouve, en premier lieu, le groupe le plus nombreux: celui des pays unilingues qui, tels le Brésil, la France, le Mexique ou les Pays-Bas, possèdent un seul système juridique. Ensuite vient le groupe plus restreint des pays où règne un bilinguisme officiel, tels le Canada, la Belgique, la Finlande et le Cameroun, voire un multilinguisme, la Suisse par exemple. Le troisième groupe, enfin, est composé d'un nombre plus restreint encore de pays où règnent à la fois un bi- ou multilinguisme et un bijuridisme, comme dans certains pays fédéraux: Belgique, Canada, Inde.

Une quatrième catégorie pourrait être envisagée, très restreinte celle-là, de pays à la fois bi- ou multilingues et bi- ou multisystémiques. Parmi les plus anciens, le Canada est de ceux-là; parmi les plus récents figure, entre autres, le Sri Lanka. Selon qu'il sera appelé à œuvrer dans tel ou tel contexte, du plus simple au plus complexe, 
le traducteur sera confronté à un facteur culturel allant du plus unifié (la France, par ex.) au moins unifié (l'Inde, par ex.), d'une culture qui se voudrait unique à une culture polymorphe. La difficulté croît à proportion du nombre de langues et de systèmes en cause. Pour ne prendre qu'un exemple du genre de difficulté qui attend le traducteur de telle ou telle catégorie, comparons deux situations juridiques, celle de la France et celle du Canada.

Pour un juriste de tradition aussi unifiée que peut l'être la branche française de la famille romano-germanique, par rapport à celle de la common law, une institution juridique étrangère ne possède qu'un équivalent dans la langue et le système d'arrivée: la notion X (par ex. : personal property) = la notion X' (meuble/bien mobilier). Mais pour un juriste de l'autre tradition, par exemple au Canada, un terme aussi banal et courant que business corporation peut avoir jusqu'à cinq équivalents normalisés (Groffier 1990), dans l'espace (Québec, Ontario, Ottawa, Nouveau-Brunswick) et dans le temps (avant ou après telle date). La tâche du traducteur n'en sera pas simplifiée.

Dans de telles conditions, où se situe pour le traducteur le plus- et le moinsdisant culturel ? Dans la culture «monochrome» des États-Unis ou de la France, par exemple, ou, peut-être, dans celle, "polychrome», du Canada, de l'Inde, du Liban ou... de Vanuatu? Le meilleur moyen de s'en assurer, nous suggère Iso Camartin, est encore de «traduire dans une autre langue ce que l'on trouve chez soi incomparable » (Camartin, 1996: 21) et d'en mesurer les résultats, "souvent accablants» (ibid.).

Pourtant, quelles que soient la méthode et la manière retenues, le but de la traduction - juridique ou autre - est d'atteindre, sinon la traduction «totale» que d'aucuns croient possible, du moins ce qu'il est convenu d'appeler l'équivalence des textes.

\section{La question de l'équivalence}

Quelle que soit la nature du texte à traduire, le principe reste le même: faire passer un message d'un texte dans un autre, de façon qu'il soit compris par la ou les personnes à qui il est destiné: le destinataire, qu'il faut préalablement déterminer. Un postulat prime en traduction "pragmatique»: seul compte le sens. Ce sens, il faut le faire comprendre au destinataire, et pour cela tous les moyens et voies appropriés sont bons.

Des sept "procédés» vus plus haut, celui de l'équivalence est sans doute celui qui a fait couler le plus d'encre. Selon les auteurs, elle sera qualifiée, entre autres, de dynamique, de fonctionnelle, de naturelle, de formelle. Elle n'en demeure pas moins le résultat le plus généralement recherché, chez les juristes comme chez les jurilinguistes (Pigeon, 1982: 271; Gémar, 1995: 142; Sarcevic, 1997: 235), quoique toujours au prix d'un compromis (Schroth, 1986: 54), toujours difficile et néanmoins nécessaire.

Toutefois, en pratique, si la question ne semble pas poser de gros problèmes au traducteur - juridique ou autre —, sur le plan théorique, en revanche, elle est loin d'avoir été tranchée puisque chaque langue «impose ses grilles aux objets du monde, en sorte que tout passage dans une autre n'est au mieux qu'une équivalence » (Hagège 1985: 49). En somme, tous domaines confondus, l'équivalence ne serait qu'un pisaller, et pour les juristes, un «compromis».

Le fond du problème toutefois réside pour moi dans cette question : une traduction (réussie, s'entend) est-elle identique à l'original, sa copie conforme, au point de 
pouvoir lui être substituée? Cette question peut sembler purement théorique puisque l'équivalence est prise comme allant de soi. En fait, pour ce qui est des textes juridiques, cette équivalence est décrétée: par la loi (l'État), par convention (entre particuliers) ou, en cas de litige, par une décision de justice. Mais de quelle équivalence parle-t-on? Est-ce que les termes suivants, jugés équivalents dans la plupart des dictionnaires et ouvrages spécialisés, s'équivalent vraiment?

\section{Rule of Law $=$ État de droit $=$ Rechtstaat}

Point n'est besoin d'une longue analyse du concept socio politique que recouvre chacun de ces termes pour se rendre compte qu'ils ne peuvent couvrir le même champ sémantique : chacun d'eux repose sur une tradition aussi ancienne que singulière. Qui plus est, «l'État de droit» des Français, en l'état actuel de la Ve République (1958), est-il le même au sein de tous les états francophones de tradition civiliste, en Belgique (état monarchique) par exemple? Et que dire du principe du rule of law entre deux pays aux institutions aussi différentes que le Royaume-Uni, qui est une monarchie parlementaire dotée d'une constitution non écrite, et les États-Unis, État fédéral reposant sur la constitution écrite la plus ancienne du monde? Ces deux termes, dont chacun porte une réalité aussi différente, peuvent-ils être traduits par «l'équivalent» allemand Rechtstaat, sinon au risque de laisser croire que l'on ignore les principes fondamentaux qui en sous-tendent les fondements?

Nous dirons, dans un tel cas et faute de mieux, que ces termes se situent sur un plan d'équivalence "fonctionnelle». La réalité que désigne chacun d'eux, soit la manière de concevoir l'État de droit, est propre à chaque pays, et donc unique dans ses modalités. Elle n'en correspond pas moins à un principe reconnu et bien établi dans les démocraties occidentales et représente de ce fait un «quasi universel» juridique. J'emploie le mot «quasi» pour montrer que le concept occidental d'État de droit ne s'étend pas à l'ensemble de la planète juridique.

Tout aussi caractéristique est le cas de good faith et de Treu und Glauben (Legrand 2000 : 111). La notion de «bonne foi » est un principe quasi universellement reconnu, mais qui ne repose pas sur les mêmes fondements juridico-socio-politiques d'un pays à l'autre. Là encore, on pourrait parler d'équivalence fonctionnelle de ces termes, même s'il n'y a pas identité de notion entre eux. Nombre de notions entrent d'ailleurs dans cette catégorie, dont celle du dol (fraud), de l'hypothèque (mortgage) et, en matière parlementaire, le Sénat. Ceux des États-Unis, du Canada et de la France, par exemple, ont peu à voir en commun.

Plus discutable, ou carrément plus simple, est le cas des termes sans équivalent d'une langue à l'autre. C'est à eux que l'on doit l'impression, si répandue dans les milieux juridiques, qu'un droit étranger est tellement différent du nôtre que la traduction en est très difficile, voire impossible (David 1974:346). En réalité, ces termes ne représentent fort heureusement qu'une infime minorité du vocabulaire juridique d'une langue et leur traitement, s'il ne pose plus de grande difficulté, reste toutefois à géométrie variable en fonction de la situation juridique et linguistique et des quatre situations possibles évoquées plus haut. Certains comparatistes, dont René David, pionnier en la matière, ont depuis longtemps suggéré de conserver tels quels en français des termes comme Common Law et Equity, qui sont des noms propres.

Cela peut convenir au lecteur d'un traité de droit comparé ou à un spécialiste de la question. Répandu dans les pays de bilinguisme et de bijuridisme - tel le Canada 
où le lecteur le moindrement informé connaît les difficultés de base tant linguistiques que juridiques - , ce procédé est sans doute moins recommandable dans une traduction dont les destinataires ignorent les subtilités des langues et des systèmes juridiques en cause, et ne saurait être utilisé sans discrimination dans toutes les situations. Mais que faire de cas tels que due process, auxquels on pourrait ajouter, par exemple, Law Lord(s), anticipatory breach of contract ou equitable interest, termes sans équivalent en français? Des notions telles que fair / fairness et reasonable, même si des équivalents fonctionnels existent, posent de sérieux dilemmes au traducteur, et cela dans nombre de langues (Fletcher 1999: 57; Sacco 1999: 169).

Une troisième situation peut être envisagée lorsqu'il n'existe pas d'équivalence fonctionnelle entre les termes, parce que les notions ne se recoupent pas (cf. Act of God et «force majeure») mais qu'il existe une traduction plus ou moins officielle ou équivalente - House of Lords / Commons / Representatives ( = chambre des Lords / des Communes / des Représentants), Trust = fiducie (Can.). D’autres situations sont possibles. Par exemple, au Canada, la traduction, dans l'Acte de l'Amérique du Nord Britannique (AANB, 1867), du mot Dominion par «Puissance» (du Canada) est un équivalent pour le moins curieux qui découle uniquement de la volonté du pouvoir politique d'imposer un terme correspondant à sa vision particulière des choses.

Quand on connaît l'étendue du champ sémantique qui sous-tend chacun de ces termes, fruits d'une longue histoire juridique et politique, croire à leur "équivalence» témoigne, au moins, d'une grande naïveté et revient à faire peu de cas des langues, cultures et traditions socio politiques de chaque pays. Ces traditions s'expriment avec force dans les méthodes et manières d'interpréter les textes, les juridiques notamment. Chaque pays appartenant à la famille et à la tradition de la common law possède sa loi d'interprétation, qui diffère souvent d'un pays et même d'une région à l'autre. Quoique l'interprétation juridique d'un texte ne relève pas de sa compétence, qui reste celle des tribunaux, ce sujet ne laissera pas le traducteur indifférent lorsqu'il s'agira d'évaluer le résultat de son travail.

\section{Équivalence linguistique des textes = équivalence juridique?}

Le problème d'équivalence juridique du texte traduit par rapport à l'original ne se pose pas dans les mêmes termes pour le traducteur et pour le juriste, le premier cherchant en principe à produire un texte équivalent et le second une équivalence juridique. Or, dans un cas comme dans l'autre, c'est la rencontre et la fusion harmonieuses des deux éléments constitutifs du texte - contenant et contenu - qui produiront l'équivalence souhaitable. Il reste à savoir ce que l'on doit entendre par "équivalence» dans la bouche d'un juriste quand un texte de droit - comme tout texte est susceptible d'interprétations différentes (Perelman 1977, Côté 1990).

Les linguistes ont avancé quelques éléments de réponse à ce problème général que pose le langage. Sur le plan juridique toutefois, de quelle équivalence parle-t-on? Peut-on, en traduction juridique, atteindre les deux objectifs à la fois, soit l'équivalence des textes dans chaque langue et dans chaque système, sans sacrifier l'un ou l'autre? Si sacrifice il y a, doit-on, en vue de réaliser l'équivalence fonctionnelle, sacrifier l'application de la règle de droit — et le but même de cette équivalence ou l'expression de la règle (Beaupré, 1987: 742) ? Tout le dilemme de la traduction juridique tient dans cette alternative fondamentale: "D'un côté, elle [l'équivalence] 
doit se garder de corrompre la langue par le calque servile qui n'en respecte pas le génie et la structure, de l'autre côté, il lui faut ne pas trahir le sens du message par l'imperfection inhérente à ce genre d'équivalence.»(Pigeon, 1982: 279).

En droit, le fond du problème est juridique: les deux textes font-ils également foi? Cette question vaut pour toute traduction juridique, que le contexte soit unilingue ou bilingue et que l'on passe ou non d'un système à un autre. Le Canadien Michael Beaupré la pose crûment : un article du Code civil du Bas-Canada qui reproduit une disposition du Code Napoléon peut-il être interprété par le canal de la version anglaise? Les tribunaux ont répondu à cette question, fort étrange pour un civiliste unilingue, mais tout à fait plausible en contexte de bilinguisme, de bijuridisme et de bisystémie, comme au Canada et au Québec (Beaupré 1986, 206). L'interprétation, dans un tel contexte, peut sembler un peu «forcée» (Sacco, 1999: 175). Il reste que le traducteur le moindrement expérimenté sait, de par sa propre interprétation du sens du texte de départ, tout ce qu'une traduction peut apporter à l'établissement du sens de ce texte, et donc à son interprétation future.

\section{Heurs et malheurs de l'équivalence}

Dans les situations juridiques où figurent deux ou plusieurs textes en regard, comme dans les pays où règne le bi- ou le multilinguisme et a fortiori dans ceux qui comptent deux systèmes juridiques différents, l'équivalence des textes est un fait légalement établi. Mais cette équivalence est-elle aussi réelle que le prétend le législateur? Nous savons que la complexité actuelle des textes juridiques, législatifs notamment, vient principalement du fait qu'ils reflètent la complexité croissante de la société qui les produit. A quoi il faut ajouter celle des systèmes juridiques. Nous savons que cette complexité s'accroît d'autant lorsque le bi- ou le multilinguisme s'additionne au bijuridisme. Mais il y a pire situation encore. Nous avons envisagé un degré supplémentaire où la complexité atteint son paroxysme lorsqu'il y a bisystémisme. C'est à ce niveau de complexité que les limites de la traduction ressortent le plus clairement.

Prenons comme exemple le cas d'un texte (code civil) rédigé, mettons, en néerlandais, traduit dans chacune des deux langues représentatives d'un grand système juridique occidental, l'anglais pour la common law et le français pour la famille romano-germanique, et supposons enfin que les trois langues soient officielles (cf. Annexe 1). De quelle équivalence textuelle, de quel système d'interprétation et de quels effets juridiques «équivalents » peut-on parler à ce propos?

Si l'on peut conclure, après lecture et analyse des trois textes, à une certaine équivalence textuelle, linguistique, voire juridique, des dispositions entre elles, il est clair que, quelque brillants que soient les analyses, commentaires, exégèses et autres gloses que peuvent produire les meilleurs comparatistes, la question de l'équivalence juridique des trois textes, à supposer qu'elle soit mise en cause, ne saurait être tranchée que par les tribunaux.

Un dernier exemple, célèbre celui-là, illustrera la portée et les limites de la traduction lorsque la puissance publique force la main du traducteur (cf. Annexe 2). Ici, la traduction semble avoir atteint ses limites quand on force la syntaxe ( from territories $=$ des territoires $)$ — et non plus seulement le lexique comme dans le cas de Dominion = Puissance - à exprimer autre chose que ce que dit le texte (Bar 1999: 313). La traduction ne produira, dans de tels cas, que le reflet renvoyé par le miroir 
déformant du langage et de l'interprétation humaine (Bourcier 1999: 376; Meyer 1999: 317).

Toutefois, et cette perspective a de quoi rassurer, lorsqu'il s'agira de produire un texte juridique à partir d'un autre texte composé en langue étrangère, parfois d'un système (juridique) différent, le traducteur est loin d'avoir dit son dernier mot (Vanderlinden 1999: 57). De la confrontation des textes sortira un tertium quid, une autre langue (Didier 1990), un hybride en somme qui n'est ni tout à fait la copie de l'original ni vraiment un texte conforme, dans la lettre comme dans l'esprit, au système d'arrivée.

\section{La lettre, l'esprit et le sens}

Alors, si le combat est inégal et l'issue certaine, faut-il se résigner au pire plutôt que de prétendre au meilleur? Non, car il est toujours possible de mieux faire, ne seraitce qu'en préparant encore mieux le traducteur aux difficultés qui l'attendent, en le formant davantage encore aux langues, systèmes et méthodes avec lesquels il est appelé à travailler. Ces solutions sont de l'ordre du possible, car, en définitive, traduire ne revient pas à trouver des équivalences aux mots, fussent-ils des "termes », comme la traductologie l'a démontré depuis des lustres, mais à exprimer dans une autre langue les idées - et, donc, le sens — porté par le texte, et cela quelle que soit la nature du texte. Les «mots» du texte de départ disparaissent pour réapparaître sous une autre forme, celle du texte d'arrivée, et dans son esprit.

Peut-être une des meilleures réponses à cette question a-t-elle été apportée par le traducteur-rédacteur de la version française du Code civil suisse, Virgile Rossel également rapporteur de langue française du projet de code adopté en 1907 et entré en vigueur en 1912 (Sarcevic 1997: 36). Selon l'article 116 de la Constitution fédérale de la Suisse, le code a été rédigé dans les trois langues officielles (allemand, français, italien), mais, en réalité, traduit de l'allemand. Le texte français a été écrit dans l'esprit du modèle jurilinguistique de l'époque, à savoir le Code Napoléon; d'où un texte coulant et idiomatique reflétant la souplesse du langage du droit qui distingue le CN. Dans un autre genre, le Code de droit canon présente aussi une traduction souple et idiomatique.

En définitive, la croyance que la traduction est chose possible repose davantage sur le principe de l'équivalence des situations que sur celui des universaux que partageraient les langues. Nous sommes encore loin de l'idéal cicéronien d'une même loi pour tous et comprise de tous. Cet idéal d'universalisme, aussi souhaitable soit-il, n'en reste pas moins encore une utopie (Legrand 2000:111) et l'exemple de la tour de Babel que constitue déjà l'Union européenne n'est pas de nature à laisser croire le contraire. La tâche du traducteur juridique, qui, déjà, n'est pas simple, ne sera pas facilitée par la diversité des situations que j'ai évoquées et qui l'attendent en cet âge de mondialisation.

Si l'on prend en compte les quatre situations juridiques et linguistiques possibles que j'ai présentées, à quoi l'on ajoutera les quatre types principaux de destinataires, auxquels viennent se greffer les trois cas d'équivalence évoqués, le traducteur de textes juridiques peut se retrouver confronté à 48 situations différentes. Et sans doute bien davantage si l'on y ajoute les méthodes et principes de traduction particuliers applicables dans chacun des cas. À chacune de ces situations le traducteur devra trouver 
une réponse adaptée au contexte (juridique, linguistique, systémique...), qui ne sera pas forcément la même d'un cas à l'autre. On est loin des sept «procédés » de traduction ou du débat sur la traduction littérale ou libre. La réalité dépasse ici largement la fiction.

En fin de compte, c'est la volonté étatique, celle de la puissance publique qui décide de l'équivalence, réelle ou supposée, de deux textes juridiques, de deux dispositions. Le traducteur, généralement seul devant son texte, ne peut compter que sur ses propres ressources pour atteindre l'équivalence recherchée. Or, en traduction juridique comme en traduction tout court, cette équivalence reste pour le moins aléatoire. C'est la conclusion qui se dégage des nombreuses études portant sur la question et que résument bien ces mots: «[... la traduction juridique ne saurait jamais être rigoureusement exacte. C'est une opération approximative, dont il convient d'apprécier la marge d'erreur. En somme, une traduction juridique constitue une simple présomption, que les intéressés doivent toujours pouvoir contester en se référant au texte authentique» (Focsaneanu 1971: 262).

Présomption est bien le mot qui ramène ce débat à sa juste proportion. C'est cette capacité de contester judiciairement la présomption que représente la traduction d'un texte qui confère à la traduction juridique un autre élément de singularité. Cela en fait une spécialité des plus difficiles à exercer (Cooray 1985: 69), car on attend du traducteur qu'il réussisse l'improbable synthèse de la lettre du droit porté par le texte et de l'esprit du système la régissant, tout en exprimant dans le texte d'arrivée le message du texte de départ selon les canons du langage du droit du destinataire. Dans la plus exigeante des hypothèses, afin d'atteindre ce but, le traducteur devrait réunir la compétence du juriste comparatiste et le savoir-faire du linguiste. S’il appartient au juge de "dire le droit», c'est bien au traducteur qu'il revient de "dire le texte».

L'activité traduisante est une réalité incontournable. L'opération traduisante, comme tout acte de langage, est une opération pour le moins approximative, voire improbable lorsque la charge culturelle du texte est lourde. Le texte juridique n'échappe pas à la malédiction de Babel et "les résultats sont souvent accablants» (Camartin 1996: 21). Lecteur peu commun et interprète naturel du sens des textes, le traducteur doit toutefois pouvoir apporter une contribution précieuse comme médiateur entre les systèmes juridiques, les langues et les cultures qu'elles véhiculent.

\section{NOTES}

1. Ma traduction de l'espagnol.

2. Pour une étude plus poussée de la question, je renvoie le lecteur au tome 2 de mon ouvrage Langage du droit et traduction, Québec, Presses de l'Université du Québec, 1995.

\section{RÉFÉRENCES}

BAR, S. (1999) : «La question des langues au sein des Nations Unies», Langues et droits, Bruxelles, Bruylant, p. 291-316.

Beaupré, M. (1987) : «La traduction juridique. Introduction», Cahiers de droit, vol. 28, Québec, Université Laval, p. 735-745.

Benvéniste, E. (1966): Problèmes de linguistique générale, Paris, Gallimard.

Berman, A. (1984): L'épreuve de l'étranger, Paris, Gallimard. 
Bourcier, D. (1999): «La novlangue du droit ou comment rendre actifs les textes juridiques", Langues et droits, Bruxelles, Bruylant, p. 369-388.

Camartin, I. (1996): Sils-Maria ou le toit du monde, Genève, Éditions Zoé (version française).

Carbonnier, J. (1995): Flexible droit, $8^{\mathrm{e}}$ éd., Paris, L.G.D.J.

Cooray, L.J.M. (1985): Changing the Language of the Law. The Sri Lanka Experience. Québec, Presses de l'Université Laval.

Cornu, G. (1990): Linguistique juridique, Paris, Montchrestien.

CôtÉ, P.-A. (1990): Interprétation des lois, $2^{\mathrm{e}}$ éd., Cowansville (Qc), Les Éditions Yvon Blais.

Crépeau, P. A. (1993) : «L'affaire Daigle et la Cour suprême du Canada ou la méconnaissance de la tradition civiliste", in Ernest CAPARros [dir.] Mélanges Germain Brière, Montréal, Wilson \& Lafleur, p. 217-281.

David, R. (1974): Les grands systèmes de droit contemporains, 6 e éd., Paris, Dalloz.

Didier, E. (1990): Langues et langages du droit, Montréal, Wilson \& Lafleur.

Dumézil, G. (1995) : Mythe et épopée I. II. III, Paris, Gallimard, coll. Quarto.

Focsaneanu, L. (1971): "Les langues comme moyen d'expression du droit international», Annuaire français de droit international, vol. 16, Paris, p. 256-263.

Gémar, J.-C. (1995): Traduire ou l'art d'interpréter, Québec, Presses de l'Université du Québec.

Groffier, E. et D. Reed (1990): La lexicographie juridique, Cowansville (Qc), Éditions Y. Blais.

HAGÈge, C. (1985): L'Homme de paroles, Paris, Fayard.

LEgrand, P. (2000) : «Brèves réflexions sur l'utopie unitaire en droit» Revue de la common law en français, vol. 3, n ${ }^{\text {os }} 1$ et 2, Moncton (N.-B.), Université de Moncton.

Lerat, P. (1995): Les langues spécialisées, Paris, PUF.

LÉvi-Strauss, C. (1949): Structures élémentaires de la parenté, Paris, PUF.

Meyer, D. (1999): «L'interprétation des traités [...]», Langues et droits, Bruxelles, Bruylant, p. 317-336.

Newmark, P. (1988): A textbook of translation, Prentice Hall Europe, Hemel Hempstead.

NidA, E. A. (1982): «Translating Legal Terms in the Bible» in J.-C. GÉmAR [dir.] Langage du droit et traduction. Essais de jurilinguistique, Montréal, Linguatech et Conseil de la langue française, p. 261-269.

- (1996): The Sociolinguistics of Interlingual Communication, Bruxelles, Les Éditions du Hazard.

PAz, O. (1983): «Lecturas y contemplaciones», Sombras de Obras, Barcelona, Seix Barral.

Perelman, C. (1977): L’empire rhétorique, rhétorique et argumentation, Paris, Librairie philosophique J. Vrin.

Pigeon, L.-P. (1982): «La traduction juridique. L'équivalence fonctionnelle» in J.-C. Gémar [dir.] Langage du droit et traduction, Québec, Conseil de la langue française, p. 271-281.

Plourde, M. [dir.] (2000): Le français au Québec. 400 ans d'histoire et de vie, Québec, Fides et les Publications du Québec.

Rouland, N. (1991): Aux confins du droit, Paris, Odile Jacob.

SACCO, R. (1999) : «Langue et droit» in R. SACCo et L. CAStellani [dir.] Les multiples langues du droit européen uniforme, Torino, L'Harmattan, p. 163-185.

SArcevic, S. (1997): New Approach to Legal Translation, The Hague, Kluwer.

Schroth, P.W (2000): «Language and Law», in Langue et droit, Bruxelles, Bruylant, p. 153-176.

Sparer, M. (1979): "Pour une dimension culturelle de la traduction juridique», Meta, 24-1, Montréal, Les Presses de l'Université de Montréal, p. 68-94.

Vanderlinden, J. (1996): Anthropologie juridique, Paris, Dalloz.

— (1999): "Langue et droit», Contemporary Law, Cowansville (Québec), Les Éditions Yvon Blais, p. 25-68.

— (1999): «Langue et droit» in Erik Jayme [dir.] : Langue et droit, Bruxelles, Bruylant, p. 65121.

Vinay, J.-P. et J. Darbelnet (1958): Stylistique comparée de l'anglais et du français, Paris, Didier.

VIAU, P. (2000) : «Quelques considérations sur la langue, le droit, le bilinguisme et le bijuridisme au Canada» Langue et droit, Bruxelles, Bruylant, p. 141-151. 


\section{ANNEXES}

ANNEXE 1

TITEL 10

ARBEIDSOVEREENKOMST

Afdeling 1

Algemene bepalingen

Art. 610. - 1. De arbeidsovereenkomst is de overeenkomst waarbij de ene partij, de werknemer, zich verbindt in dienst van de andere partij, de werkgever, tegen loon gedurende zekere tijd arbeid te verrichten.

- 2. Indien een overeenkomst zowel aan de omschrijving van lid 1 voldoet als aan die van een andere door de wet geregelde bijzondere soort van overeenkomst, zijn de bepalingen van deze titel en de voor de andere soort van overeenkomst gegeven bepalingen naast elkaar van toepassing. In geval van strijd zijn de bepalingen van deze titel van toepassing.
TITLE 10

\section{CONTRACT OF EMPLOYMENT}

\section{Section 1}

General provisions

Art. 610. - 1. A contract of employment is a contract whereby one party - the employee - undertakes to perform work in the service of the other party - the employer - for remuneration during a given period.

-2 . If a contract of employment fulfils both the definition of paragraph 1 and that of another special type of contract regulated by law, the provisions of this Title and the provisions governing the other type of contract apply in conjunction. In the event of a conflict, the provisions of this Title apply.

\section{TITRE DIXIÈME DU CONTRAT DE TRAVAIL}

Section première Dispositions générales

Art. 610. - 1. Le contrat de travail est celui par lequel une personne, le salarié, s'oblige, pour un temps limité et moyennant salaire, à effectuer du travail pour le compte d'une autre personne, l'employeur.

-2. Si un contrat satisfait aussi bien à la description du paragraphe premier qu'à celle d'une autre espèce particulière de contrat régi par la loi, les dispositions du présent titre ainsi que celles régissant cette autre espèce de contrat lui sont simultanément applicables. En cas de conflit, les dispositions du présent titre s'appliquent.

\section{ANNEXE 2}

(i) Withdrawal of Israel armed forces from territories occupied in the recent conflict; i) Retrait des forces israéliennes des territoires occupés lors du récent conflit;

Source: United Nations Resolutions 